Article

\title{
Study on the Stability of DeoxyArbutin in an Anhydrous Emulsion System
}

\section{Chih-Chien Lin ${ }^{1}{ }^{1 *}$, Chao-Hsun Yang ${ }^{1}$, Nai-Fang Chang ${ }^{1}$, Pey-Shiuan Wu ${ }^{1}$, Yi-Shyan Chen ${ }^{1}$, Shu-Mei Lee ${ }^{2}$ and Chiu-Wen Chen ${ }^{1}$}

1 Department of Cosmetic Science, Providence University, 200 Chung-Chi Road, Shalu, Taichung 43301, Taiwan; E-Mails: chyang@pu.edu.tw (C.-H.Y.); nfchang@pu.edu.tw (N.-F.C.); jwu2@pu.edu.tw (P.-S.W.); yishyan@gm.pu.edu.tw (Y.-S.C.); rita770115@hotmail.com (C.-W.C.)

2 Department of Cosmetic Science and Management, Mackay Medicine, Nursing and Management College, 92 Shengjing Road, Beitou, Taipei 11260, Taiwan; E-Mail: s107@eip.mkc.edu.tw

* Author to whom correspondence should be addressed; E-Mail: chchlin@pu.edu.tw; Tel.: +886-4-26328001 ext. 15409; Fax: +886-4-26311167.

Received: 3 August 2011; in revised form: 1 September 2011 / Accepted: 7 September 2011 / Published: 15 September 2011

\begin{abstract}
The skin-whitening agent, deoxyArbutin, is a potent tyrosinase inhibitor that is safer than hydroquinone and arbutin. However, it is thermolabile in aqueous solutions, where it decomposes to hydroquinone. Pharmaceutical and cosmetic emulsions are normally oil-in-water $(\mathrm{o} / \mathrm{w})$ or water-in-oil (w/o) systems; however, emulsions can be formulated with no aqueous phase to produce an anhydrous emulsion system. An anhydrous emulsion system could offer a stable vehicle for compounds that are sensitive to hydrolysis or oxidation. Therefore, to enhance the stability of deoxyArbutin in formulations, we chose the polyol-in-silicone, anhydrous emulsion system as the basic formulation for investigation. The quantity of deoxyArbutin and the accumulation of hydroquinone in both hydrous and anhydrous emulsions at various temperatures were analyzed through an established high performance liquid chromatographic (HPLC) method. The results indicated that water increased the decomposition of deoxyArbutin in the formulations and that the polyol-in-silicone, oil-based, anhydrous emulsion system provided a relatively stable surrounding for the deoxyArbutin that delayed its degradation at $25{ }^{\circ} \mathrm{C}$ and $45^{\circ} \mathrm{C}$. Moreover, the composition of the inner hydrophilic phase, containing different amounts of glycerin and propylene glycol, affected the stability of deoxyArbutin.
\end{abstract}


Thus, these results will be beneficial when using deoxyArbutin in cosmetics and medicines in the future.

Keywords: anhydrous emulsion system; deoxyArbutin; hydroquinone; skin whitening; stability

\section{Introduction}

Tyrosinase is an enzyme with a copper center that is widely expressed in many life forms and is mainly involved in the formation of pigments, such as melanin and other polyphenolic compounds. The biosynthesis of melanin in melanocytes might be affected by cellular tyrosinase activity [1,2]. In addition, increased production and accumulation of melanin leads to many hyperpigmentation diseases such as melasma, solar lentigines, and post-inflammatory hyperpigmentation. Therefore, investigation into depigmentation mechanisms and the clinical aspects of skin whitening agents is very important $[3,4]$. Various compounds have demonstrated inhibitory effects on melanogenesis through inhibition of the enzymatic activity of tyrosinase [5-7]. For example, hydroquinone (Figure 1A) and deoxyArbutin (dA; Figure 1B) are both potent skin-whitening agents that are capable of suppressing the function of tyrosinase. DeoxyArbutin (4-[(tetrahydro-2H-pyran-2-yl)oxy]phenol) was first shown by Boissy et al. to exhibit greater inhibition of tyrosinase activity and to be safer than hydroquinone and arbutin [8]. Moreover, deoxyArbutin demonstrated fast and persistent skin lightening effects both in an animal model and in a human trial $[9,10]$. However, in our previous study, we found that this skin whitening agent was thermolabile in aqueous solutions and decomposes to hydroquinone under these conditions [11]. Instability in water posed developmental and practical problems for using deoxyArbutin in cosmetics and medication. Thus, enhancing the stability of this skin-whitening agent is important for its development.

Figure 1. Chemical structure of hydroquinone (A) and deoxyArbutin (B).

(A)<smiles>Oc1ccc(O)cc1</smiles>

(B)<smiles>Oc1ccc(OC2CCCCO2)cc1</smiles>

Pharmaceutical and cosmetic emulsions are normally oil-in-water (o/w) or water-in-oil (w/o) systems (Figure 2A). However, emulsions can be formulated with no aqueous phase to produce an anhydrous, non-aqueous, oil-in-oil or oil-polar solvent emulsion system [12]. A phospholipid-based [13], petroleum-formamide/dimethyl formamide/dimethyl sulfoxide-based [14], hydrocarbon-formamidebased [15] or silicone-based [12] system could offer a stable vehicle for transporting active compounds that are sensitive to hydrolysis or oxidation. For instance, a polyol-in-silicone anhydrous emulsion 
system comprised of a polar phase with polyol, which replaces water, and a nonpolar phase with silicone oil (Figure 2B) could be used in this manner. Specifically, the polyol in similar systems has regularly been selected as propylene glycol, butylene glycol or glycerin.

Figure 2. Illustration of a normal emulsion (A) and an anhydrous emulsion (B).

(A)

normal emulsion

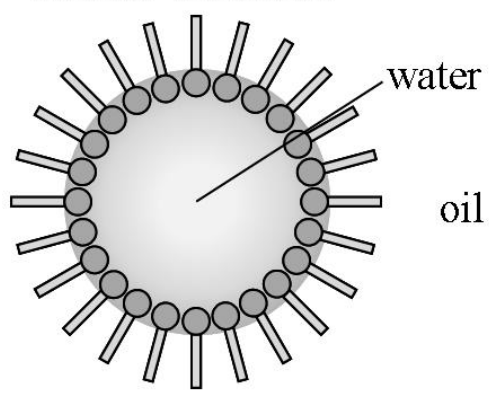

(B)

\section{anhydrous emulsion}

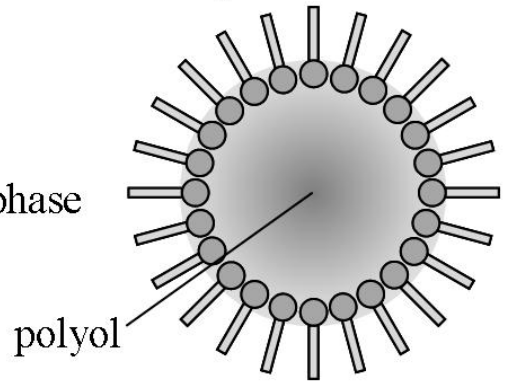

$\Longleftarrow$ Surfactant

To enhance the stability of deoxyArbutin in formulations, we chose the polyol-in-silicone anhydrous emulsion system as the basic formulation for investigation. The quantity of deoxyArbutin in both a normal (hydrous) emulsion and an anhydrous emulsion at various temperatures were analyzed through an established high performance liquid chromatographic (HPLC) method. Moreover, the accumulation of hydroquinone in these formulations were analyzed and compared to each other.

\section{Results and Discussion}

The major objective of this work was to examine the stability of deoxyArbutin in an anhydrous emulsion system. Therefore, in the first part of the study, we used two polyol-in-silicone anhydrous emulsions in formulations containing deoxyArbutin. Secondly, we used an established HPLC method to confirm the quantity of deoxyArbutin and hydroquinone in these formulations at various temperatures. Thus, the difference in the stability of deoxyArbutin in these normal and anhydrous emulsions will be revealed by this study.

\subsection{Preparation of DeoxyArbutin-Containing Formulations}

The compositions of the anhydrous (anH-1/2) and hydrous (H-1/2) formulations used in this study are listed in Table 1 . We used $4 \%$ or $2 \%$ cetyl dimethicone copolyol as the emulsifier to formulate the silicone-based formulations 1 (anH-1/H-1) and 2 (anH-2/H-2), respectively. The outer phase, or oil phase, was produced by adding cyclomethicone with or without stearyl dimethicone and isostearyl isostearate. Additionally, the inner phase, or hydrophilic phase, was composed of deoxyArbutin (3\%), propylene glycol and glycerin, with or without water. In this phase, propylene glycol and glycerin provide the solubility of deoxyArbutin. In addition, anhydrous (anH-1/2) and hydrous (H-1/2) formulations were defined according to the water content of hydrophilic phase in formulations. Thus, we prepared two polyol-in-oil anhydrous emulsions and two water-in-oil (w/o) hydrous emulsions for this study (Table 1). 
Table 1. Compositions of formulations used in this study.

\begin{tabular}{ccccccccc}
\hline & \multicolumn{7}{c}{ ingredients } \\
\cline { 2 - 8 } formulation & dA & $\begin{array}{c}\text { cetyl dimethicone } \\
\text { copolyol }\end{array}$ & cyclomethicone & $\begin{array}{c}\text { stearyl } \\
\text { dimethicone }\end{array}$ & $\begin{array}{c}\text { isostearyl } \\
\text { isostearate }\end{array}$ & $\begin{array}{c}\text { propylene } \\
\text { glycol }\end{array}$ & $\begin{array}{c}\text { glycerin } \\
\text { deionized } \\
\text { water }\end{array}$ \\
\hline anH-1 & $3 \%$ & $4 \%$ & $17 \%$ & $0 \%$ & $0 \%$ & $46 \%$ & $30 \%$ & $0 \%$ \\
H-1 & $3 \%$ & $4 \%$ & $17 \%$ & $0 \%$ & $0 \%$ & $46 \%$ & $10 \%$ & $20 \%$ \\
anH-2 & $3 \%$ & $2 \%$ & $14 \%$ & $3 \%$ & $2 \%$ & $70 \%$ & $6 \%$ & $0 \%$ \\
H-2 & $3 \%$ & $2 \%$ & $14 \%$ & $3 \%$ & $2 \%$ & $50 \%$ & $6 \%$ & $20 \%$ \\
\hline
\end{tabular}

anH: anhydrous emulsion, $\mathrm{H}$ : hydrous emulsion.

The prepared formulations had no obvious difference in appearance between each other. All prepared formulations were centrifuged at $2330 \times \mathrm{g}$ for $20 \mathrm{~min}$ to check the stability of the formulations. The results indicated that the formulations made in this study were stable (no phase separation occurs) and remained stable until the experiment was completed (14 weeks) and after the formulations were incubated at $45^{\circ} \mathrm{C}$ (data not shown).

\subsection{Stability of DeoxyArbutin in Formulations at Low Temperature}

To verify the stability of deoxyArbutin in an anhydrous emulsion system, we placed the prepared formulations at various temperatures and then analyzed the amount of deoxyArbutin and hydroquinone in each formulation on each of the selected days for a total of 98 days (14 weeks). In all of the formulations, concentrations of deoxyArbutin were slightly decreased during the time course at $4{ }^{\circ} \mathrm{C}$, and the lowest amount of deoxyArbutin, in the H-2 formulation on the 98th day, was $86.08 \pm 1.97 \%$ (Figure 3A). Notably, hydroquinone was not detectable (N.D.) in any of the formulations at any time point at $4{ }^{\circ} \mathrm{C}$, except for the $\mathrm{H}-2$ formulation on the 98 th day $(2.41 \pm 0.84 \%$; Figure $3 \mathrm{~B})$.

Figure 3. Stability of deoxyArbutin (A) and the accumulation of hydroquinone (B) at $4{ }^{\circ} \mathrm{C}$ (low temperature) in various formulations.
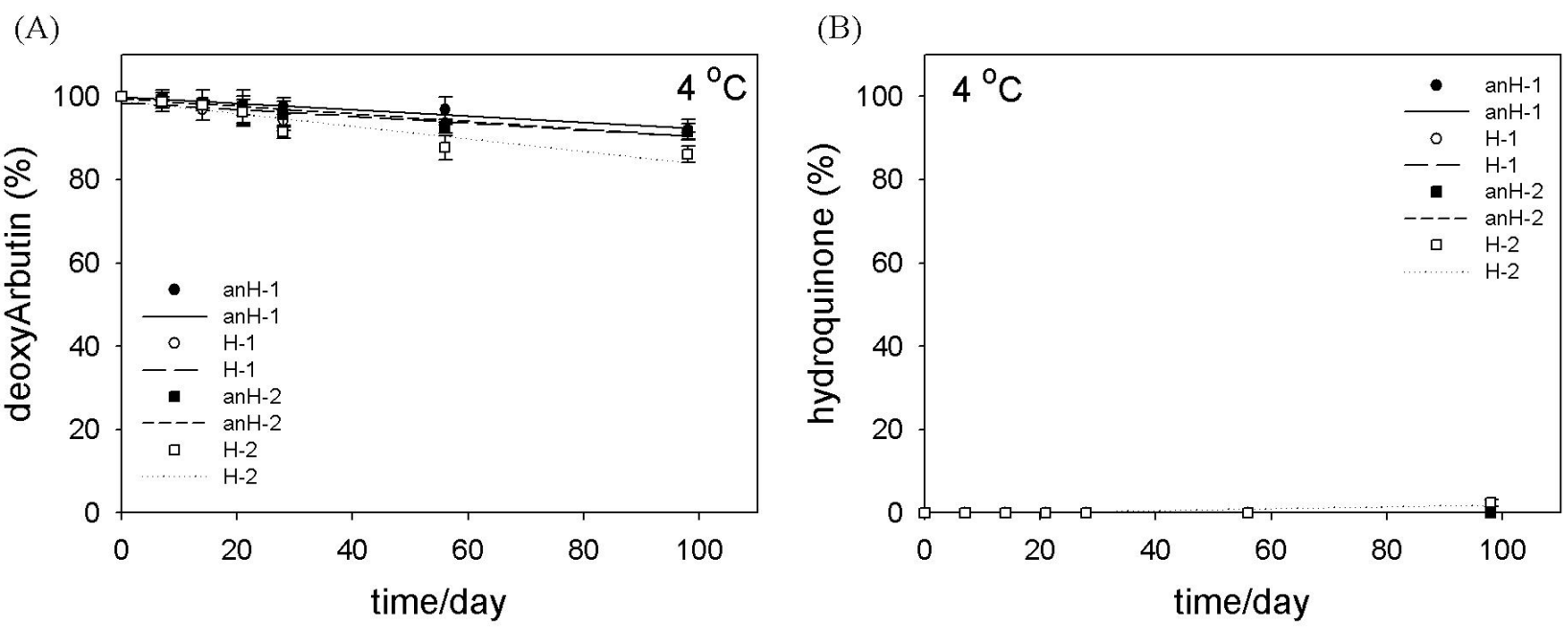

Although the difference in the stability of deoxyArbutin in the normal and anhydrous emulsions was not evident at $4{ }^{\circ} \mathrm{C}$, there was a slight increase in the stability of deoxyArbutin when the 
formulation was prepared without water (Figure 3A). However, this result also indicated that formulated deoxyArbutin kept at a low temperature underwent degradation.

\subsection{Stability of DeoxyArbutin in Formulations at Moderate Temperature}

At $25{ }^{\circ} \mathrm{C}$, the amount of deoxyArbutin in both anhydrous formulations (anH-1/2) was higher than that in the hydrous formulations (H-1/2; Figure 4A). On the last day of this experiment, the retention percentages of deoxyArbutin in the anH-1 and anH-2 formulations were $76.86 \pm 4.11 \%$ and $87.29 \pm 4.63 \%$, respectively. Comparatively, the retention percentage of deoxyArbutin in the $\mathrm{H}-1$ and H-2 formulations on the 98th day were only $26.49 \pm 4.51 \%$ and $40.28 \pm 3.07 \%$, respectively; thus, we concluded that deoxyArbutin in an anhydrous emulsion system was more stable. In addition, the accumulation of hydroquinone in these formulations was inversely correlated with the decay of deoxyArbutin, such as in formulation $\mathrm{H}-1$, which had the lowest retention percentage of deoxyArbutin and the most abundant hydroquinone accumulation (Figure 4A and 4B). This decomposition process in aqueous solutions had been demonstrated in our previous study [11].

Figure 4. Stability of deoxyArbutin (A) and the accumulation of hydroquinone (B) at $25{ }^{\circ} \mathrm{C}$ (moderate temperature) in various formulations.
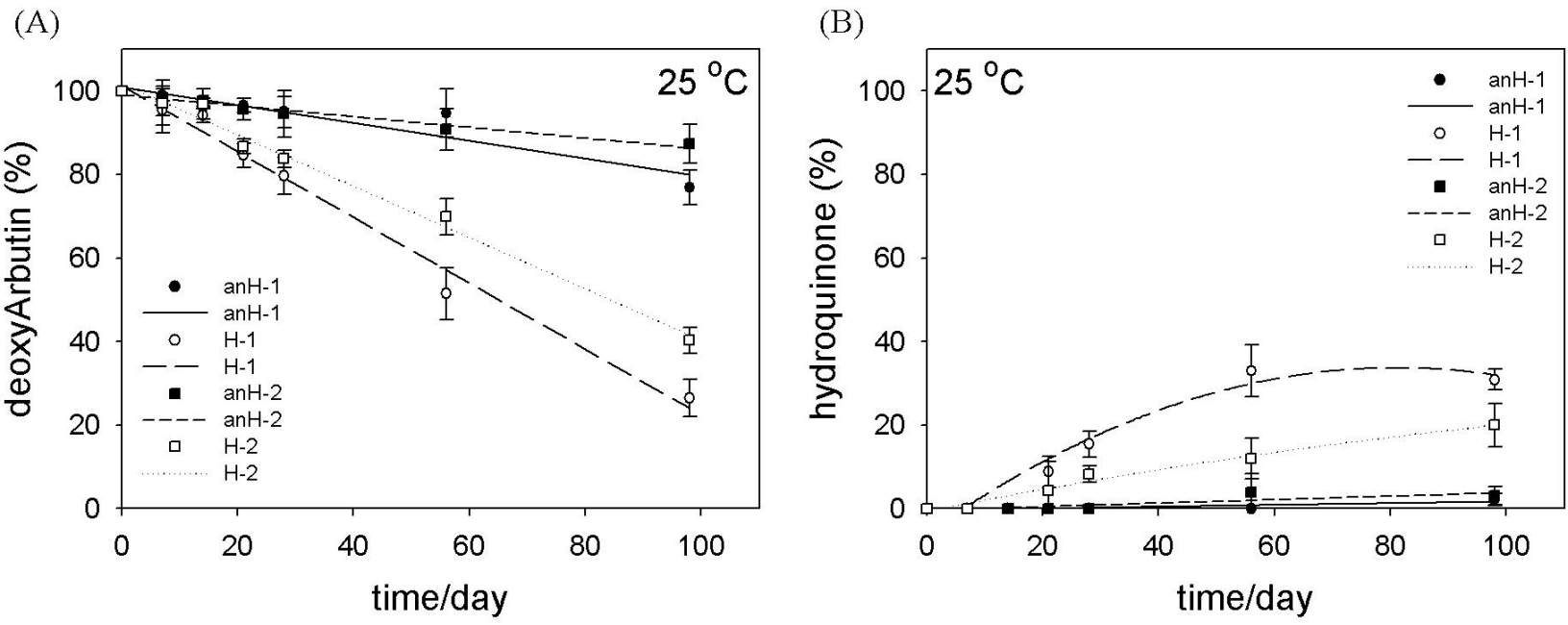

Moreover, deoxyArbutin was more stable in a water or polyol-in-silicone oil emulsion at $25{ }^{\circ} \mathrm{C}$ (higher than $84.62 \%$ in all formulations on the 21 st day) than what was seen in our previous study where deoxyArbutin had been dissolved in water with $10 \%$ propylene glycol $(49.42 \%)$ [11]. This result indicated that the droplet structures of emulsions provided a stable environment for deoxyArbutin and that a similar phenomenon of enhanced drug stability was revealed in numerous emulsion systems [16-18].

\subsection{Stability of DeoxyArbutin in Formulations at High Temperature}

At $45{ }^{\circ} \mathrm{C}$ and in all formulations, deoxyArbutin decomposed quickly (Figure 5A); however, the most unstable surroundings were the water-containing formulations (H-1/2). Moreover, deoxyArbutin was completely decayed within two weeks in the $\mathrm{H}-1$ and $\mathrm{H}-2$ formulations at $45^{\circ} \mathrm{C}$, but deoxyArbutin in the anH-1 and anH-2 formulations persisted for approximately 40 to 50 days (Figure $5 \mathrm{~A}$ ). These 
results demonstrated that deoxyArbutin in an anhydrous emulsion system was more stable than in a hydrous emulsion system. Furthermore, hydroquinone accumulated in all formulations when maintained at this temperature (Figure 5B). Although the percentage of hydroquinone was decreased in the high temperature environment at the end of this experiment, the relationship between deoxyArbutin decomposition and hydroquinone accumulation was still evident (Figure 5A and 5B). In addition, hydroquinone at high temperature may also undergo some decomposition process. Thus, the content of hydroquinone was decreased after a period of $40-60$ days at $45^{\circ} \mathrm{C}$ (Figure 5B).

Figure 5. Stability of deoxyArbutin (A) and the accumulation of hydroquinone (B) at $45^{\circ} \mathrm{C}$ (high temperature) in various formulations.
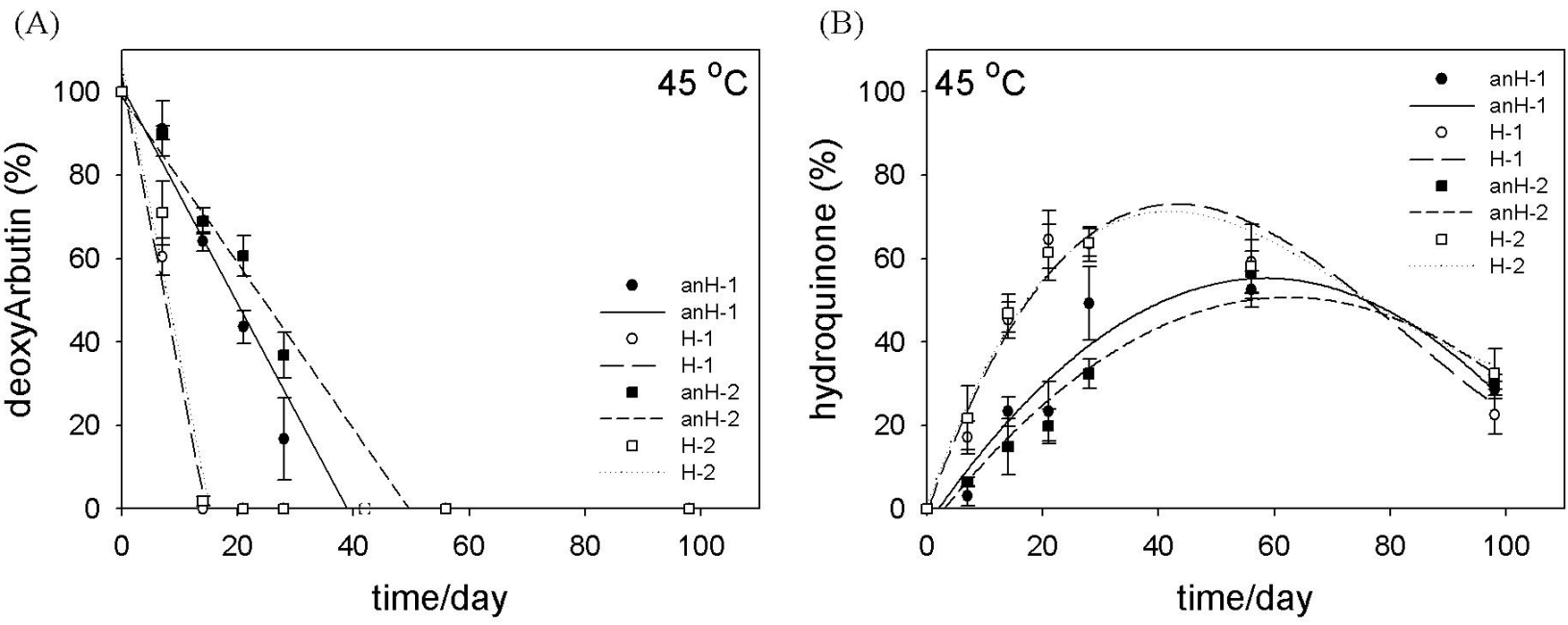

Furthermore, when comparing formulations 1 and 2, the retention percentage of deoxyArbutin in $\mathrm{H}-2$ was higher than in $\mathrm{H}-1$ at $25{ }^{\circ} \mathrm{C}$ (Figure 4A), and the retention percentage of deoxyArbutin in anH-2 was higher than that in anH-1 at $45{ }^{\circ} \mathrm{C}$ (Figure 5A). This consequence possibly resulted from the diverse compositions of formulations 1 and 2. For instance, formulation 1 had a higher amount of glycerin and a lower amount of propylene glycol (Table 1). This difference provided diverse hydroxyl groups in the inner hydrophilic phase of these two types of formulations. Furthermore, the difference in the composition of the oil phase could have also presented a minor effect on the stability of deoxyArbutin in these formulations (Table 1).

In summary, water may increase the decomposition of deoxyArbutin in formulations; thus, the anhydrous emulsion system could provide a relatively stable surrounding that could delay degradation of deoxyArbutin at temperatures of 25 and $45{ }^{\circ} \mathrm{C}$. The results from this study are important for improving the formulation for deoxyArbutin. Besides, many factors such as $\mathrm{pH}$ value or other ingredients in the emulsions also affect the stability of deoxyArbutin. Moreover, the adding of stabilizer or antioxidant into the formulation or with oil-in-oil formulations may further enhance the stability of deoxyArbutin. In addition, these strategies can also apply to other compounds that are sensitive to hydrolysis. We are currently investigating these potential methods to improve the applications of deoxyArbutin. 


\section{Experimental Section}

\subsection{Materials}

DeoxyArbutin (99.9\%) was purchased from Denjelly Co., Ltd (Miaoli, Taiwan, R.O.C), and hydroquinone (99.8\%) was purchased from Wako Pure Chemical Industries (Osaka, Japan). HPLC-grade methanol was purchased from Merck (Darmstadt, Germany), cetyl dimethicone copolyol and stearyl dimethicone were purchased from EVONIK Goldschmidt GmbH (Essen, Germany), cyclomethicone was purchased from Dow Corning Corporation (Midland, MI, USA), and isostearyl isostearate was purchased from Corum Inc. (Taipei, Taiwan, R.O.C). Propylene glycol, glycerin and other chemicals were purchased from Sigma-Aldrich (St. Louis, MO, USA), and deionized distilled water $\left(\mathrm{ddH}_{2} \mathrm{O}\right)$, which was used for solutions, was obtained with a Milli-Q system (Millipore, Bedford, MA, USA).

\subsection{Preparation of Formulations and Emulsion Stability Test}

The compositions of the formulations, including the hydrous and anhydrous emulsions, are listed in Table 1. DeoxyArbutin (3\%) was first pre-dissolved in propylene glycol, and all formulations were prepared by premixing the hydrophilic and hydrophobic phases. After premixing, the hydrophilic and hydrophobic components were emulsified via a high-shear mixer at an agitation speed of $200 \mathrm{rpm}$ for $2 \mathrm{~min}$. Subsequently, increase the agitation speed at $1000 \mathrm{rpm}$ for $5 \mathrm{~min}$ to complete the emulsification. To test the stability of emulsions, all prepared formulations were centrifuged at 2,330 $\mathrm{g}$ for $20 \mathrm{~min}$ to check their stability. Besides, at every time point, the formulations were also determined whether the phase separation occurs to confirm the emulsions are stable.

\subsection{Sample Preparation and High Performance Liquid Chromatography (HPLC) Analysis}

For sample preparation, $0.25 \mathrm{~g}$ of emulsion was dissolved in $50 \mathrm{~mL}$ of methanol and then sonicated for $30 \mathrm{~min}$ in a water bath sonicator. The extracted sample was subsequently diluted (1:24 (v/v)) for analysis in pure methanol. The analytic procedures for deoxyArbutin and hydroquinone were performed according to our previous study [11]. Specifically, each $20-\mu \mathrm{L}$ sample was injected into the HPLC apparatus (Agilent 1100 series, USA), which utilized a pre-packed C18 reversed-phase column (Mightysil RP-18, GP 250-4.6, Kanto Chemical, Tokyo, Japan). The mobile phase, composed of methanol and water $(60: 40(\mathrm{v} / \mathrm{v}), \mathrm{pH} 7)$, was run at a flow rate of $1 \mathrm{~mL} / \mathrm{min}$ The solvents were filtered separately through a $0.45 \mathrm{~mm}$ filter (Millipore) and mixed in the desired proportions. The UV wavelength was set to $280 \mathrm{~nm}$ for detection of both deoxyArbutin and hydroquinone, and the ratios of the compound peak areas to the internal standard peak areas were calculated using the corresponding concentrations. These ratios were then used to obtain the calibration graph. In the determination of hydroquinone, the percentage of hydroquinone was calculated by the molar ratio of deoxyArbutin to hydroquinone, i.e., $3 \mathrm{~g}$ of deoxyArbutin can completely decompose to $1.701 \mathrm{~g}$ of hydroquinone. 


\subsection{Stability Studies of DeoxyArbutin in the Emulsion System}

The normal and anhydrous formulations containing 3\% deoxyArbutin were placed in glass bottles and kept in the dark. Once in an incubator, these samples were exposed to the following temperatures: $4{ }^{\circ} \mathrm{C}$ (low temperature), $25{ }^{\circ} \mathrm{C}$ (moderate temperature) and $45{ }^{\circ} \mathrm{C}$ (high temperature). At each of the designated time points, the formulations were analyzed using the previously described HPLC method to monitor the stability of deoxyArbutin and hydroquinone in these emulsion systems.

\subsection{Statistical Analysis}

All data were obtained in three independent experiments and then analyzed to determine the mean values. Statistical comparisons of the means and simple correlation coefficients were performed using the Student's $t$-test.

\section{Conclusions}

In conclusion, we have demonstrated that water may enhance the decomposition of deoxyArbutin in formulations and that a polyol-in-silicone oil-based anhydrous emulsion system can provide a relatively stable surrounding that delays the degradation of deoxyArbutin at temperatures of 25 and $45^{\circ} \mathrm{C}$. Moreover, the composition of the inner hydrophilic phase, which contains a different amount of glycerin and propylene glycol, might affect the stability of deoxyArbutin. Therefore, the results from this study can help in determining practical uses for deoxyArbutin in cosmetics and medicines in the future.

\section{Acknowledgments}

The authors are grateful for financial support from the National Science Council of the Republic of China (R.O.C.) awarded to C.-C. Lin (NSC99-2313-B-126-002-MY3).

\section{References}

1. Huang, H.C.; Chiu, S.H.; Chang, T.M. Inhibitory effect of [6]-GINGEROL ON MELANOgenesis in B16F10 melanoma cells and a possible mechanism of action. Biosci. Biotechnol. Biochem. 2011, 75, 1067-1072.

2. Kubo, I.; Nitoda, T.; Nihei, K. Effects of quercetin on mushroom tyrosinase and B16-F10 melanoma cells. Molecules 2007, 12, 1045-1056.

3. Chang, T.S. An updated review of tyrosinase inhibitors. Int. J. Mol. Sci. 2009, 10, 2440-2475.

4. Fais, A.; Corda, M.; Era, B.; Fadda, M.B.; Matos, M.J.; Quezada, E.; Santana, L.; Picciau, C.; Podda, G.; Delogu, G. Tyrosinase inhibitor activity of coumarin-resveratrol hybrids. Molecules 2009, 14, 2514-2520.

5. Dawley, R.M.; Flurkey, W.H. 4-hexylresorcinol, a potent inhibitor of mushroom tyrosinase. J. Food Sci. 1993, 58, 609-610.

6. Sato, K.; Toriyama, M. Depigmenting effect of catechins. Molecules 2009, 14, 4425-4432. 
7. Tai, S.S.; Lin, C.G.; Wu, M.H.; Chang, T.S. Evaluation of depigmenting activity by 8-hydroxydaidzein in mouse B16 melanoma cells and human volunteers. Int. J. Mol. Sci. 2009, $10,4257-4266$.

8. Hamed, S.H.; Sriwiiyanont, P.; Wickett, R.R.; Boissy, R. Effect of deoxyarbutin on melanogenesis: In vivo comparison with other melanogenesis inhibitor. J. Cosmet. Sci. 2004, 55, 118-119.

9. Boissy, R.E.; Visscher, M.; DeLong, M.A. DeoxyArbutin: A novel reversible tyrosinase inhibitor with effective in vivo skin lightening potency. Exp. Dermatol. 2005, 14, 601-608.

10. Chawla, S.; de Long, M.A.; Visscher, M.O.; Wickett, R.R.; Manga, P.; Boissy, R.E. Mechanism of tyrosinase inhibition by deoxyArbutin and its second-generation derivatives. Br. J. Dermatol. 2008, 159, 1267-1274.

11. Yang, C.-H.; Chen, Y.-S.; Lai, J.-S.; Hong, W.W.L.; Lin, C.-C. Determination of the thermodegradation of deoxyArbutin in aqueous solution by high performance liquid chromatography. Int. J. Mol. Sci. 2010, 11, 3977-3987.

12. Suitthimeathegorn, O; Jaitely, V.; Florence, A.T. Novel anhydrous emulsions: Formulation as controlled release vehicles. Int. J. Pharm. 2005, 298, 367-371.

13. Wang, T.; Wang, N.; Hao, A.; He, X.; Li, T.; Deng, Y. Lyophilization of water-in-oil emulsions to prepare phospholipid-based anhydrous reverse micelles for oral peptide delivery. Eur. J. Pharm. Sci. 2010, 39, 373-379.

14. Cameron, N.R.; Sherrington, D.C. Non-aqueous high internal phase emulsions. Preparation and stability. J. Chem. Soc. Faraday Trans. 1996, 92, 1543-1547.

15. Sakthivel, T.; Jaitely, V.; Patel, N.V.; Florence, A.T. Non-aqueous emulsions: Hydrocarbonformamide systems. Int. J. Pharm. 2001, 214, 43-48.

16. Shibata, H.; Nakagawa, S.; Tsutsumi, Y. Optimization of protein therapies by polymer-conjugation as an effective DDS. Molecules 2005, 10, 162-180.

17. Chae, G.S.; Lee, J.S.; Kim, S.H.; Seo, K.S.; Kim, M.S.; Lee, H.B.; Khang, G. Enhancement of the stability of BCNU using self-emulsifying drug delivery systems (SEDDS) and in vitro antitumor activity of self-emulsified BCNU-loaded PLGA wafer. Int. J. Pharm. 2005, 301, 6-14.

18. Heiati, H.; Tawashi, R.; Phillips, N.C. Drug retention and stability of solid lipid nanoparticles containing azidothymidine palmitate after autoclaving, storage and lyophilization. J. Microencapsul. 1998, 15, 173-184.

(C) 2011 by the authors; licensee MDPI, Basel, Switzerland. This article is an open access article distributed under the terms and conditions of the Creative Commons Attribution license (http://creativecommons.org/licenses/by/3.0/). 